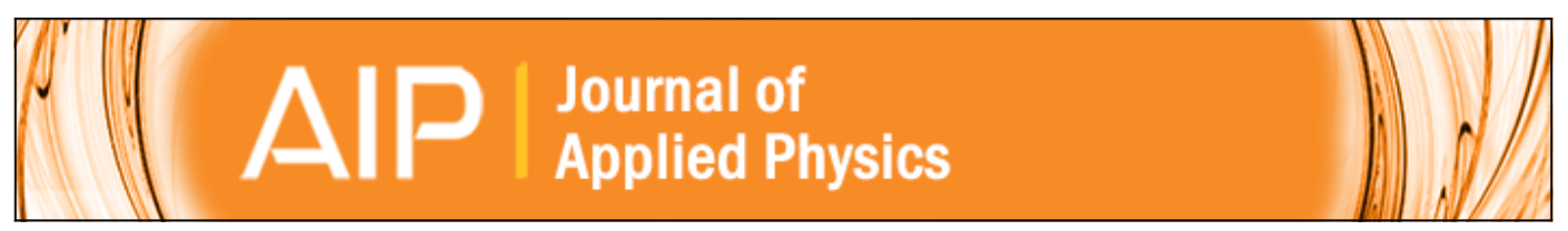

\title{
Hybrid stretchable circuits on silicone substrate
}

A. Robinson, A. Aziz, Q. Liu, Z. Suo, and S. P. Lacour

Citation: Journal of Applied Physics 115, 143511 (2014); doi: 10.1063/1.4871279

View online: http://dx.doi.org/10.1063/1.4871279

View Table of Contents: http://scitation.aip.org/content/aip/journal/jap/115/14?ver=pdfcov

Published by the AIP Publishing

\section{Articles you may be interested in}

Monolithic integration of silicon CMOS and GaN transistors in a current mirror circuit

J. Vac. Sci. Technol. B 30, 02B101 (2012); 10.1116/1.3665220

Preface to Special Topic: Selected Papers from the International Conference on Flexible and Printed Electronics, Jeju Island, Korea, 2009

J. Appl. Phys. 108, 102701 (2010); 10.1063/1.3510244

Complementary metal oxide silicon integrated circuits incorporating monolithically integrated stretchable wavy interconnects

Appl. Phys. Lett. 93, 044102 (2008); 10.1063/1.2963364

Hybrid carbon nanotube-silicon complementary metal oxide semiconductor circuits

J. Vac. Sci. Technol. B 25, 2577 (2007); 10.1116/1.2800322

Stretchable gold conductors on elastomeric substrates

Appl. Phys. Lett. 82, 2404 (2003); 10.1063/1.1565683

\section{AlP Re-register for Table of Content Alerts}




\title{
Hybrid stretchable circuits on silicone substrate
}

\author{
A. Robinson, ${ }^{1, a)}$ A. Aziz, ${ }^{1, b)}$ Q. Liu, ${ }^{2}$ Z. Suo, ${ }^{2}$ and S. P. Lacour $\left.{ }^{3, c}\right)$ \\ ${ }^{1}$ Nanoscience Centre, University of Cambridge, Cambridge CB01FF, United Kingdom \\ ${ }^{2}$ School of Engineering and Applied Sciences and Kavli Institute for Bionano Science and Technology, \\ Harvard University, Cambridge, Massachusetts 02138, USA \\ ${ }^{3}$ Centre for Neuroprosthetics and Laboratory for Soft Bioelectronics Interfaces, School of Engineering, \\ Ecole Polytechnique Fédérale de Lausanne, Lausanne 1015, Switzerland
}

(Received 26 February 2014; accepted 1 April 2014; published online 11 April 2014)

\begin{abstract}
When rigid and stretchable components are integrated onto a single elastic carrier substrate, large strain heterogeneities appear in the vicinity of the deformable-non-deformable interfaces. In this paper, we report on a generic approach to manufacture hybrid stretchable circuits where commercial electronic components can be mounted on a stretchable circuit board. Similar to printed circuit board development, the components are electrically bonded on the elastic substrate and interconnected with stretchable electrical traces. The substrate-a silicone matrix carrying concentric rigid disks - ensures both the circuit elasticity and the mechanical integrity of the most fragile materials. (C) 2014 AIP Publishing LLC. [http://dx.doi.org/10.1063/1.4871279]
\end{abstract}

\section{INTRODUCTION}

Stretchable electronics is a fast emerging field that provides radically new form factors to electronic circuits. ${ }^{1}$ Some of the most exciting applications include novel human-machine interfaces in the form of skin-like ${ }^{2-6}$ or epidermal circuitry, ${ }^{7,8}$ and bioelectronic monitoring. ${ }^{9}$ The main requirement for stretchable circuits is that the electronic devices and circuit performance do not, or only predictably, change with the applied mechanical loading.

One approach to manufacture stretchable circuits is to produce a stretchable circuit board onto which standard, offthe-shelf components may be mounted. ${ }^{10,11}$ Rigid component islands are distributed on the surface of an elastomeric substrate and interconnected with elastic wiring. This hard-onsoft integration however suffers from large mechanical strain concentration at the interface between the rigid (printed circuit board PCB-like) islands and the stretchable substrate. Because interconnects must run across these interfaces, early electrical failure of the stretchable circuit is often observed. ${ }^{12}$

This paper presents a simple solution to this challenge and demonstrates a functional hybrid stretchable circuit fabricated on an engineered stretchable circuit board. The latter is designed so that non-deformable regions are embedded within the elastomeric substrate (rather than on top of the substrate), and the strain within the elastic wiring, i.e., the top surface strain, smoothly increases across the rigid-toelastic interface with the applied strain and does not exceed the applied strain. Non-deformable regions are prepared by embedding two concentric disks of stiff plastic foil within an elastomer matrix. We demonstrate the dual disks design is a straightforward way to keep the strain above the smaller disk to $0 \%$ and suppress the strain peak across the hard-soft

\footnotetext{
${ }^{a)}$ Current address: Nokia Research Centre, Cambridge, United Kingdom. Electronic mail: adam.1.robinson@nokia.com

${ }^{b)}$ Current address: Physics Department, University of Lancaster, Lancaster, United Kingdom. Electronic mail: a.aziz1@lancaster.ac.uk

c) Author to whom correspondence should be addressed. Electronic mail: stephanie.lacour@epfl.ch
}

transitions. We monitor the surface strain within elastic wiring prepared with stretchable thin gold film interconnects ${ }^{13}$ and highlight minimal mechanical fatigue in the metallic conductors upon cyclic stretching to $20 \%$ uni-axial strain. Packaged components, i.e., an operational amplifier (Op Amp), LED, and resistors, are subsequently mounted and interconnected on the stretchable circuit board to form an oscillating circuit, which frequency is designed to linearly decrease with the applied strain. The electromechanical integrity of the circuit is maintained over repeated stretch cycling demonstrating the potential of our stretchable circuit boards.

\section{OVERVIEW OF THE HYBRID STRETCHABLE CIRCUIT BOARD}

Stretchable substrates may be formed as (i) a homogeneous elastomer membrane, (ii) a mechanically graded elastomer, or (iii) an elastomeric membrane carrying embedded rigid platforms. A bulk elastomer membrane stretches uniformly so large strains appear across its surface. Macroscopic strain applied across a mechanically graded elastomer (prepared, for example, with photo-patternable $\mathrm{PDMS}^{14}$ ) is distributed through the polymer so that the stiffest, patterned regions stretch less than the softest ones. Yet, truly rigid regions, i.e., non-stretchable, cannot be prepared with this approach. Embedding stiff platforms within the thickness of the stretchable substrate can provide "zerostrain" zones on the surface of the substrate. ${ }^{12,15}$ The strain profile on the surface of such engineered substrates may present a sharp peak close to the edge of the platform and the effective "zero-strain" surface area depends strongly on the geometry and density of the embedded rigid platforms material. Here, we propose the use of concentric rigid disks embedded in the elastomeric substrate to maximize the nostrain surface area and modulate further the surface strain across the soft-hard boundary.

Figure 1 presents a schematic cross-section of the hybrid stretchable circuit board. It consists of a millimetre thick 


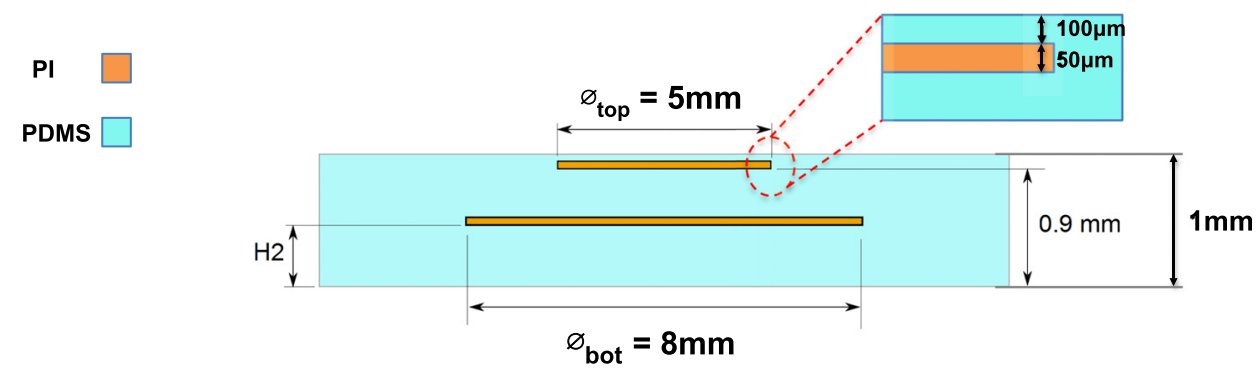

FIG. 1. Schematic cross section of the double-disk stretchable substrate.

PDMS substrate within which two concentric discs of polyimide (PI) foil (50 $\mu \mathrm{m}$ thick) are embedded. The upper disk is the smallest and is positioned towards the PDMS surface. The second disk has a bigger diameter than that of the first one and is embedded deeper in the PDMS membrane. The top disk ensures the strain at the PDMS surface immediately above it is pinned to zero while the bottom disk guarantees the surface strain to be zero across the complete surface area of the small disk ${ }^{12}$ and suppresses any sharp peak strain across the non-deformable to deformable surface. ${ }^{15}$

We compare the effects of embedding one disk and two disks by using a combination of finite element simulations and experimental measurements.

Let us first consider embedding a single disk close to the surface of the substrate. Since the plastic disk is much stiffer than the elastomeric substrate, such a construct is highly non-symmetric. When the substrate is stretched, the thin disk bends into a concave shape (Fig. 2(a)). Far away from the disk, the substrate becomes flat. To make a transition from the concave shape above the disk to the flat shape far away from the disk, the substrate bends into a convex shape right off the edge of the disk. Consequently, the convex region of the substrate develops a large strain (Fig. 2(b)). This high strain may cause failure of the interconnects. ${ }^{12}$

To reduce the strain on the surface of the substrate, we embed a second, larger, disk, roughly at a depth about half of the thickness of the substrate. Because this large disk is placed in the mid-plane of the substrate, when the substrate is stretched, the two disks no longer bend significantly (Fig. 2(c)). Consequently, the surface of the substrate above the edge of the bottom disk no longer develops a high peak of strain (Fig. 2(d)). Furthermore, because the top disk is embedded close to the surface of the substrate, the strain on the surface of the substrate rapidly decays near the edge of the top disk, giving a large area for active devices. The strains determined from the finite element simulation agree well with experimental data. The strain in the two-disk case is much smaller than that in the single-disk case (Figs. 2(b) and 2(d)).

We further illustrate our concept with experimental recording of the strain across the surface of the substrate (Figs. 2(b) and 2(d)). We introduce an ordered matrix of PDMS microposts distributed all over the top PDMS substrate. The PDMS substrate was moulded against a silicon wafer patterned with cylindrical holes, $2 \mu \mathrm{m}$ deep and $2 \mu \mathrm{m}$ in diameter, arranged in a square array with a pitch of $12 \mu \mathrm{m}$. These microposts are small enough compared to the thick PDMS substrate not to affect the mechanical properties at its surface. They are used as visual markers to measure the local surface strain of the PDMS. While stretching, the distance variation between the microposts on the PDMS surface was measured with an optical microscope, and the local strain profile along the stretch direction was calculated. Each strain value was calculated by measuring the distance between ten adjacent pillars. We assumed the error in determining the position of the edge of each micropost should be approximately the same as the micropost diameter $(2 \mu \mathrm{m})$, giving $\pm 1 \mu \mathrm{m}$ for each micropost and a total of $\pm 2 \mu \mathrm{m}$ for the row. The error was then calculated assuming a $\pm 2 \mu \mathrm{m}$ uncertainty for each strain value.

Figures 2(b) and 2(d) display the recorded strain across the single and double disk sample when a $20 \%$ strain is applied along the $\mathrm{x}$-axis. The double disk sample is made of (a)

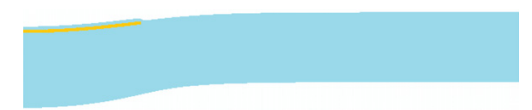

(b)

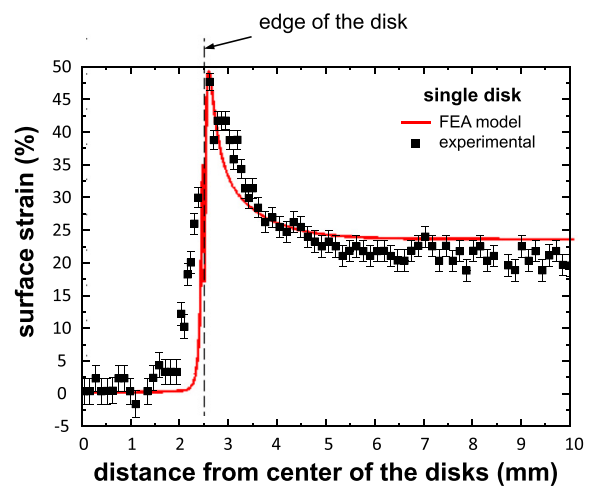

(c)

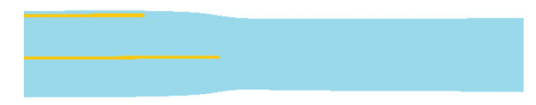

(d)

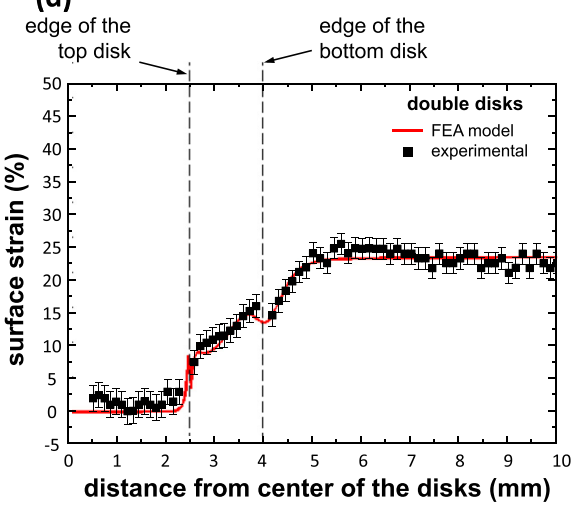

FIG. 2. Surface strain profiles across the non-deformable to deformable interface when the substrate is stretched to $20 \%$ strain. (a) and (c) are the deformations of the finite element simulation in the case of a single disk and double disks embedded within the elastomer. The top disk is $100 \mu \mathrm{m}$ from the surface and the bottom disk is half way through the substrate. The top and bottom disks are $5 \mathrm{~mm}$ and $8 \mathrm{~mm}$ in diameter, respectively. The scale in vertical direction is magnified 50\%. (b) and (d) Solid lines are the surface strain calculated from the finite element simulations. Data points are the experimentally measured data. 
(a)
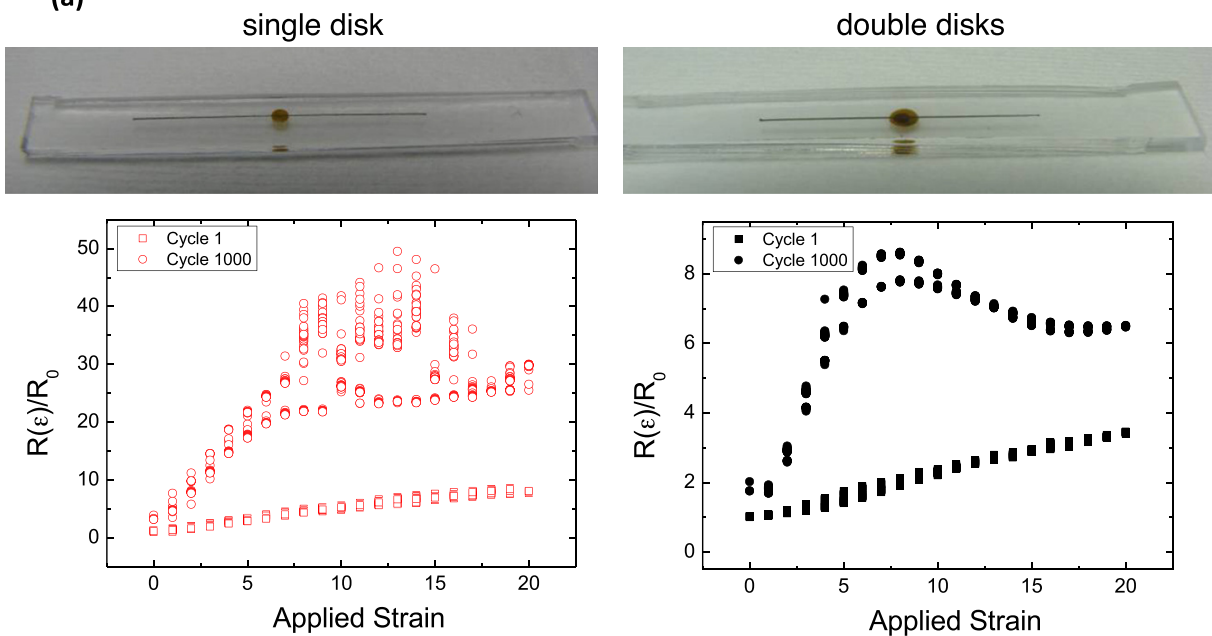

(b)
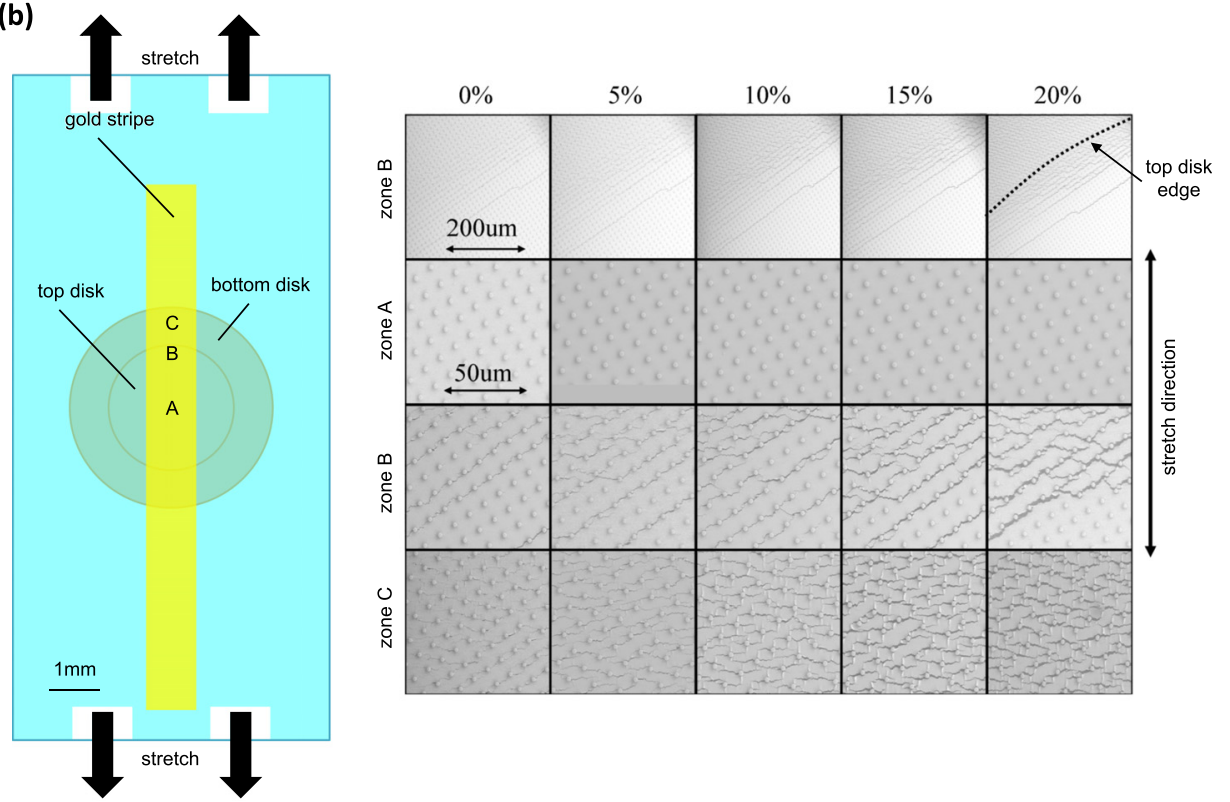

FIG. 3. Stretchable interconnects. (a) Optical micrographs of a stretchable metallic stripe patterned above a single disk (left) and double disk (right) embedded in the silicone substrate. Corresponding electrical resistance change as a function of applied macroscopic strain and cycle number. (b) Series of scanning electron micrographs taken across the interconnect surface as a function of the applied strain and location above the rigid disks. a $5 \mathrm{~mm}$ diameter PI disk embedded $100 \mu \mathrm{m}$ below the top surface and a second PI disk ( $8 \mathrm{~mm}$ diameter) embedded at $\mathrm{H} 2=0.5 \mathrm{~mm}$. The single disk sample has the $5 \mathrm{~mm}$ diameter disk embedded $100 \mu \mathrm{m}$ below the top surface. The experimental data concur with the finite element model. At $20 \%$ applied strain, a large peak reaching $\sim 40 \%$ is observed at the edge of the single disk while it is suppressed in the case of the double disk design. Furthermore, the strain is locked at $0 \%$ across the complete surface of the top disk and the strain increases from $0 \%$ to $20 \%$ in the region above the second larger disk is very gradual. Vertical lines mark the position of the edge of the $5 \mathrm{~mm}$ and $8 \mathrm{~mm}$ diameter disks. Disks of this size were studied as they provide a rigid region large enough to hold a standard rigid electrical component. The strains determined from the finite element simulation remarkably agree with the experimental data.

\section{ELECTRICAL INTERCONNECTS}

Thin-metal film interconnects are patterned on the top surface of the hybrid stretchable substrate and define a stretchable electrical network between the non-deformable areas of the substrate. Their electromechanical performance is presented Figure 3(a) as a function of applied strain and for both substrate designs. Gold thin-film stripes were evaporated on top of the single and double disk substrate, and their electrical resistance was recorded as a function of applied strain and number of cycles. The interconnect is a $2 \mathrm{~mm}$ wide and $1.5 \mathrm{~cm}$ long stripe of $\mathrm{Cr}(5 \mathrm{~nm}) / \mathrm{Au}(50 \mathrm{~nm})$ bi-layer evaporated on the PDMS substrate through a shadow mask.

Both substrates can carry highly stretchable interconnects but interestingly the double disk design allows for a much smaller change in resistance with cycling. After 1000 cycles, the interconnect resistance of the stripe running above the double disk design increases by a factor of 7 (at 20\% applied strain), compared to a factor 30 for the single disk design. This suggests reduced mechanical damage in the gold thin film on the double disk design. Note that the evolution of the $\mathrm{R}$ (strain) profile with cycling from a triangular to a box-like profile is typical of evaporated thin gold film on PDMS. ${ }^{13}$

We further observed the surface of the gold interconnect patterned on a "double disk" substrate using scanning 
(a)

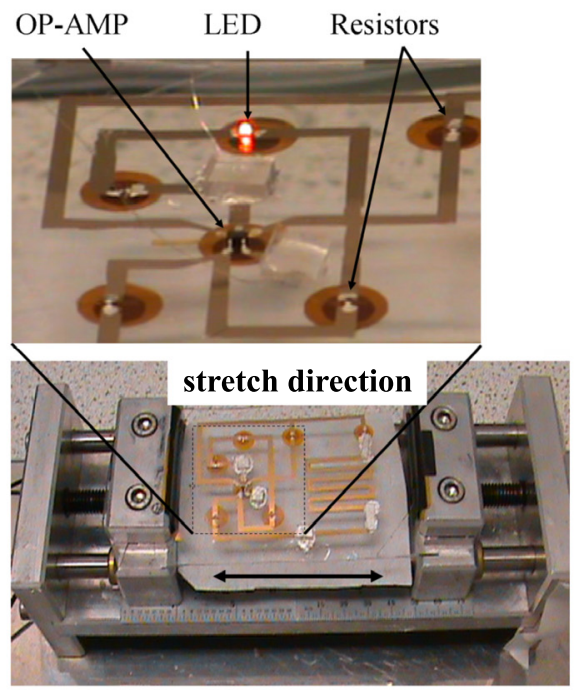

(b)

(c)
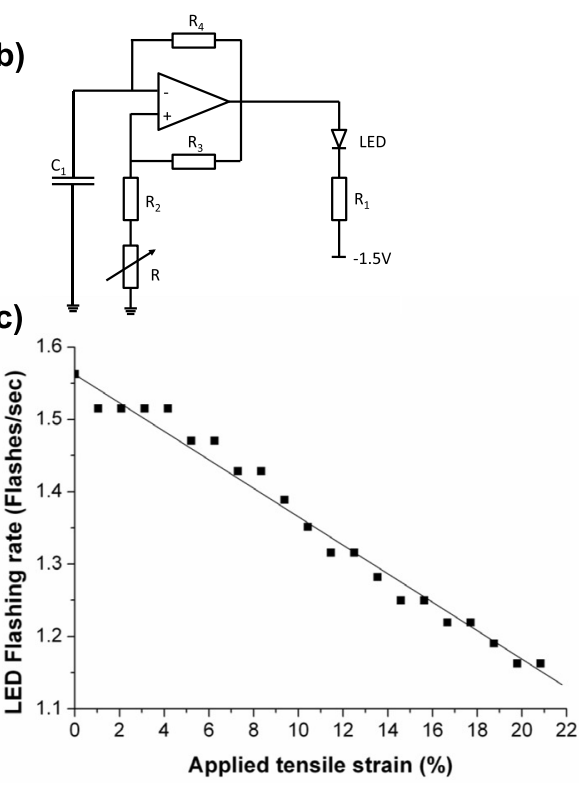

FIG. 4. Hybrid stretchable circuit. (a) Pictures of the hybrid multivibrator prepared on the stretchable circuit board. The larger polyimide disks are 8 $\mathrm{mm}$ in diameter. Close up of some components (top); circuit mounted in the uniaxial stretcher (bottom). (b) Electronic diagram of the multivibrator circuit. (c) Flashing rate of the LED as a function of applied uniaxial strain. electron microscopy. We monitored its topography as a function of applied strain in a FEI/Philips XL30 Environmental Scanning Electron Microscope (ESEM). For ease of surface tracking, the top surface of the PDMS is covered with the same PDMS microposts as described above. Figure 3(b) shows a series of micrographs taken above the rigid platform, at the edge of the $5 \mathrm{~mm}$ diameter disk and on the stretchable PDMS region after 1000 stretch cycles to $20 \%$ strain. Row (a) displays the gold surface at the edge of the 5 $\mathrm{mm}$ disk at $0 \%, 5 \%, 10 \%, 15 \%$, and $20 \%$ strain. The dotted line indicates the approximate position of the edge of $5 \mathrm{~mm}$ diameter disk. Row (b) shows the gold surface above the rigid region. There is no sign of strain or cracking in the gold film, even after 1000 stretch cycles. Row (c) shows the highest strain regions around the edge of the smaller disk. A gradual increase in the size of the cracks with increasing strain is observed. After about a thousand stretch cycles, these cracks may merge to a length that may be as long as the width of the gold stripe leading to electrical failure. The nature of the cracks' progression at the disk edge is different than that of the cracks on the region above the bigger disk, where they originate at the pillars but terminate after a short distance between the pillars, as shown in row (d). Poisson compression, normal to the stretch direction, can also be very clearly seen at $10 \%, 15 \%$, and $20 \%$ stretch in row (d), and results into a small buckling the gold layer originating from the microposts. The wrinkles run from one post to the immediately adjacent one. Investigation of series of ESEM images shows however that the main mechanical failure still occurs at the edge of the smaller disk but its occurrence is delayed thanks to the presence of the larger PI disk.

\section{STRETCHABLE HYBRID CIRCUITS}

A multivibrator circuit with a flashing LED was then implemented combining the stretchable circuit board and discrete electronic components (Fig. 4). The circuit is operating so that the rate of a flashing LED decreases when the circuit is stretched. The oscillating frequency is defined as

$$
f=\frac{1}{2 R_{4} C_{1} \ln \left(\frac{1+\beta}{1-\beta}\right)} \quad \text { with } \quad \beta=\frac{R_{2}+R}{R_{2}+R+R_{3}},
$$

where $\mathrm{R}_{2}, \mathrm{R}_{3}, \mathrm{R}_{4}$, and $\mathrm{C}_{1}$ are discrete resistors and capacitance while $R$ is a variable serpentine resistance patterned with stretchable thin gold film on the PDMS substrate.

The stretchable circuit board includes 7 double disks areas distributed across the stretchable elastomer matrix to host each of the 7 discrete components (4 resistors, 1 capacitance, and 1 OpAmp). Stretchable thin gold film interconnects are patterned on the top surface of the elastomer membrane to complete the circuit. The components are positioned above the "rigid" areas and electrically connected with the stretchable wiring using silver paste.

When the circuit is stretched, the resistance of the meander is increased, decreasing the flashing rate of the LED. Graph $4 \mathrm{c}$ shows the flashing rate of the LED, which linearly decreases with the applied tensile strain. The stretchable resistance increases by about a factor of 4 when stretched to $20 \%$ strain leading to a frequency decrease from 1.55 to 1.15 , corresponding to the theoretical variation of $f$.

\section{CONCLUSION}

This paper provides a solution to a very common problem faced in fabricating stretchable circuits. When nonstretchable regions are created in a stretchable media, for example, PDMS, high strain regions are created while stretching. For practical circuits, electrical wiring between components must run across the stretchable and nonstretchable regions, where large tensile strain may occur and lead to poor reliably of the electrical circuit and early electromechanical failure. The "double disk" design proposed in this paper provides a simple yet efficient strategy to integrate disparate electrical components on a stretchable substrate. The proposed stretchable circuit boards will allow for straightforward manufacturing of stretchable electronics. 


\section{ACKNOWLEDGMENTS}

This work was supported by the UK Engineering and Physical Sciences Research Council-EPSRC, the Bertarelli Foundation, and a European Research Council Starting Grant, ERC, No. 259419. Q.L. and Z.S. acknowledge the support of the National Science Foundation Materials Research Science and Engineering Center.

\section{APPENDIX: MATERIALS AND METHODS}

\section{Stretchable circuit board fabrication}

First, a $100 \mu \mathrm{m}$ thick layer of PDMS was spin-coated on a silanized Si substrate and cured at $65^{\circ} \mathrm{C}$ for $24 \mathrm{~h}$. A polyimide disk (Kapton $\mathrm{HN}, 50 \mu \mathrm{m}$ thick, $5 \mathrm{~mm}$ diameter) was then attached using a small amount of un-crosslinked PDMS and cured. The surface energy of the PDMS was increased using a $30 \mathrm{~s}$ exposure to the ozone plasma and a second layer of PDMS ( $0.4 \mathrm{~mm}$ thick) was cast on the wafer and cured. These processing steps were repeated for embedding the larger diameter disk (Kapton HN, $50 \mu \mathrm{m}$ thick, $8 \mathrm{~mm}$ diameter). Once the PDMS was completely crosslinked, the substrate was carefully peeled-off the Si wafer and inverted.

Stretchable interconnects were prepared by evaporation through a polyimide foil mask patterns of thin gold films $(\mathrm{Cr}$ adhesive layer, $5 \mathrm{~nm}$, Au layer $50 \mathrm{~nm}$ ) and gentle release of the mask. The surface of the double-disk substrate was perfectly smooth; no defects or catastrophic cracking was observed in the thin gold features.

\section{Stretchable hybrid circuit assembly}

Discrete, off-the-shelf electronic components were positioned manually directly onto the stretchable circuit board. Contact pads on the stretchable board were designed slightly larger than the corresponding pads on the components. Soft silver paste (Epotek H27D) ensured reliable electrical bonding between the rigid components and the stretchable interconnects. The hybrid circuit presented Fig. 4 included resistances, R1, R2, R3, and $\mathrm{R} 4$ of $270 \Omega, 1 \mathrm{~K} \Omega, 1.5 \mathrm{~K} \Omega$, and $330 \mathrm{~K} \Omega$, respectively, a $1 \mu \mathrm{F}$ capacitance and a MCP3001 operational amplifier driving the LED.

\section{Silicone micropost surfaces}

Inverted micropost molds were produced on silicon wafers using positive photoresist (AZ5214e, MicroChemicals) patterned by photolithography. The silicon wafers were first dehydrated at $200{ }^{\circ} \mathrm{C}$ and coated with an adhesive layer of hexamethyldisilazane (HMDS). The photoresist was spin coated at $2000 \mathrm{rpm}$ for $30 \mathrm{~s}$ and prebaked for $2 \mathrm{~min}$ at $110^{\circ} \mathrm{C}$ to obtain a $2 \mu \mathrm{m}$ thick film. After UV exposure through a chromium mask in a KarlSuss MJB3 mask aligner, the pattern was developed in a 1:4 developer (MIF351B, MicroChemicals) to deionized (DI) water mixture then rinsed in deionized water. The patterned wafers were subsequently perflurorinated and coated with liquid PDMS (Dow Corning, Sylgard 184, 10:1 polymer:crosslinker ratio). The elastomer was then cured in air at $65^{\circ} \mathrm{C}$ for $24 \mathrm{~h}$ and released from the wafer.

\section{Simulation details}

The finite element simulation presented in this work is accomplished through the commercial software ABAQUS. In order to better compare with the experimental data in Figure 2(c), the geometry adopts the post-experiment measurement of the cross section, where the total thickness is 1 $\mathrm{mm}$, the top disk is buried $100 \mu \mathrm{m}$ from the top surface, the bottom disk is $0.5 \mathrm{~mm}$ from the bottom surface. Both the disk and the substrate are modeled as neo-hookean material. The shear modulus ratio is taken to be 2000:1 between polyimide and PDMS. The bulk modulus is assumed to be three orders of magnitude higher than the shear modulus so that the material is almost incompressible. Since the radius of curvature of the disks is much larger than the thickness of the substrate, all the simulation is done in plane strain condition, which saves time significantly. From the good agreement between experiments and simulation, such an approximation is satisfactory. The strain over the disks not only depends on the loading strain and the geometry of the disks themselves but also the spacing between the disks. So for simplicity purpose, in simulations we match the far field equilibrium strain as the boundary condition.

${ }^{1}$ T. Someya, Stretchable Electronics (Wiley, 2013).

${ }^{2}$ T. Someya, Y. Kato, T. Sekitani, S. Iba, Y. Noguchi, Y. Murase, H. Kawaguchi, and T. Sakurai, Proc. Natl. Acad. Sci. U. S. A. 102(35), $12321-12325$ (2005).

${ }^{3}$ B. C.-K. Tee, C. Wang, R. Allen, and Z. Bao, Nat. Nanotechnol. 7, 825-832 (2012).

${ }^{4}$ S. P. Lacour, I. M. Graz, S. Bauer, and S. Wagner, in IEEE Engineering in Medicine and Biology Conference (IEEE, Boston, 2011), pp. 8373-8376.

${ }^{5}$ M. L. Hammock, A. Chortos, B. C.-K. Lee, J. B.-H. Tok, and Z. Bao, Adv. Mater. 25, 5997-6038 (2013).

${ }^{6}$ M. Kaltenbrunner, T. Sekitani, J. Reeder, T. Yokota, K. Kuribara, T. Tokuhara, M. Drack, R. Schwödiauer, I. Graz, S. Bauer-Gogonea, S. Bauer, and T. Someya, Nature 499, 458-463 (2013).

${ }^{7}$ D.-H. Kim, N. Lu, R. Ma, Y.-S. Kim, R.-H. Kim, S. Wang, J. Wu, S. M. Won, H. Tao, A. Islam, K. J. Yu, T.-I. Kim, R. Chowdhury, M. Ying, L. Xu, M. Li, H.-J. Chung, H. Keum, M. McCormick, P. Liu, Y.-W. Zhang, F. G. Omenetto, Y. Huang, T. Coleman, and J. A. Rogers, Science 333, 838-843 (2011)

${ }^{8}$ J.-W. Jeong, W.-H. Yeo, A. Akhtar, J. J. S. Norton, Y.-J. Kwack, S. Li, S.Y. Jung, Y. Su, W. Lee, J. Xia, H. Cheng, Y. Huang, W.-S. Choi, T. Bretl, and J. A. Rogers, Adv. Mater. 25, 6839-6846 (2013).

${ }^{9}$ D.-H. Kim, N. Lu, Y. Huang, and J. A. Rogers, MRS Bull. 37, 226-235 (2012).

${ }^{10}$ J. Vanfleteren, M. Gonzalez, F. Bossuyt, Y.-Y. Hsu, T. Vervust, I. D. Wolf, and M. Jablonski, MRS Bull. 37, 254-260 (2012).

${ }^{11}$ F. Bossuyt, T. Vervust, and J. Vanfleteren, IEEE Trans. Compon., Packag., Manuf. Technol. 3(2), 229-235 (2013).

${ }^{12}$ A. Romeo, Q. Liu, Z. Suo, and S. P. Lacour, Appl. Phys. Lett. 102(13), 131904 (2013).

${ }^{13}$ I. Graz, D. Cotton, and S. P. Lacour, Appl. Phys. Lett. 94, 071902 (2009).

${ }^{14}$ D. P. J. Cotton, A. Popel, I. M. Graz, and S. P. Lacour, J. Appl. Phys. 109, 054905 (2011).

${ }^{15}$ I. Graz, D. Cotton, A. Robinson, and S. P. Lacour, Appl. Phys. Lett. 98, 124101 (2011). 